University of Montana

ScholarWorks at University of Montana

Biomedical and Pharmaceutical Sciences

Faculty Publications

Biomedical and Pharmaceutical Sciences

2019

\title{
MODELING OF EXCITATORY AMINO ACID TRANSPORTERS AND CLEARANCE OF SYNAPTIC CLEFT ON MILLISECOND TIME SCALE
}

\author{
Denis M. Shchepakin \\ University of Montana - Missoula \\ Leonid Kalachev \\ University of Montana, Missoula \\ Michael Kavanaugh \\ University of Montana - Missoula
}

Follow this and additional works at: https://scholarworks.umt.edu/biopharm_pubs

Part of the Medical Sciences Commons, and the Pharmacy and Pharmaceutical Sciences Commons Let us know how access to this document benefits you.

\section{Recommended Citation}

Shchepakin, Denis M.; Kalachev, Leonid; and Kavanaugh, Michael, "MODELING OF EXCITATORY AMINO ACID TRANSPORTERS AND CLEARANCE OF SYNAPTIC CLEFT ON MILLISECOND TIME SCALE" (2019). Biomedical and Pharmaceutical Sciences Faculty Publications. 29.

https://scholarworks.umt.edu/biopharm_pubs/29

This Article is brought to you for free and open access by the Biomedical and Pharmaceutical Sciences at ScholarWorks at University of Montana. It has been accepted for inclusion in Biomedical and Pharmaceutical Sciences Faculty Publications by an authorized administrator of ScholarWorks at University of Montana. For more information, please contact scholarworks@mso.umt.edu. 


\title{
MODELING OF EXCITATORY AMINO ACID TRANSPORTERS AND CLEARANCE OF SYNAPTIC CLEFT ON MILLISECOND TIME SCALE
}

\author{
Denis Shchepakin ${ }^{1,2, *},{\text { LeOnid } \text { Kalachev }^{1} \text { and Michael Kavanaugh }}^{2}$
}

\begin{abstract}
Excitatory Amino Acid Transporters (EAATs) operate over wide time scales in the brain. They maintain low ambient concentrations of the primary excitatory amino acid neurotransmitter glutamate, but they also seem to play a significant role in clearing glutamate from the synaptic cleft in the millisecond time-scale process of chemical communication that occurs between neurons. The detailed kinetic mechanisms underlying glutamate uptake and clearance remain incompletely understood. In this work we used a combination of methods to model EAAT kinetics and gain insight into the impact of transport on glutamate dynamics in a general sense. We derive reliable estimates of the turnover rates of the three major EAAT subtypes expressed in the mammalian cerebral cortex. Previous studies have provided transporter kinetic estimates that vary over an order of magnitude. The values obtained in this study are consistent with estimates that suggest the unitary transporter rates are approximately 20 -fold slower than the time course of glutamate in the synapse. A combined diffusion/transport model provides a possible mechanism for the apparent discrepancy.
\end{abstract}

Mathematics Subject Classification. 92C45, 35K57, 41A60.

Received November 2, 2018. Accepted April 17, 2019.

\section{INTRODUCTION}

Neurons and surrounding glial cells are the primary functional and structural units of central and peripheral nervous systems. A neuron is an electrically excitable cell that receives, integrates, and transmits information by a combination of electrical and chemical neurotransmitter signaling. Rapid synaptic transmission involves release of neurotransmitter from presynaptic neuron terminals followed by diffusion across a synaptic gap and binding to postsynaptic receptors that transduce the chemical signal into an electrical signal in the postsynaptic cell (see Fig. 1). Functions of glial cells include support and homeostatic maintenance of the neurons' environment, and in some cases, reuptake of neurotransmitter.

In mammalian brain, excitatory synaptic transmission is mediated predominantly by the neurotransmitter glutamate. In this work we focus on the transport and diffusion mechanisms involved in controlling glutamate concentration changes during the signaling process. Data from a number of studies is consistent with the idea that glutamate transporters maintain low ambient concentrations to prevent persistent glutamate receptor activation

Keywords and phrases: Chemical-kinetics, asymptotic analysis, diffusion, glutamate transport.

1 Department of Mathematical Sciences, University of Montana, Missoula, MT, USA.

2 Department of Biomedical Pharmaceutical Sciences, University of Montana, Missoula, MT, USA.

* Corresponding author: denis.shchepakin@umconnect.umt.edu 


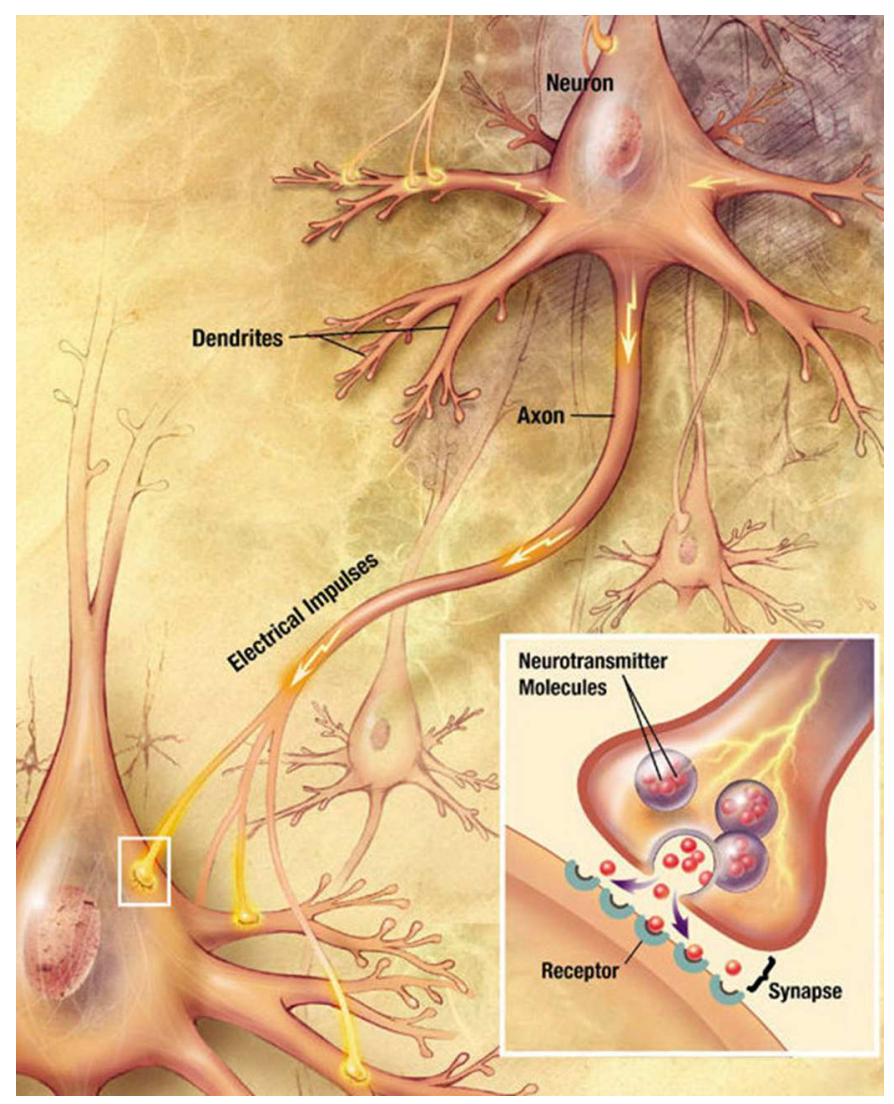

Figure 1. Cartoon representation of neurons and a neuronal transmission (Wikimedia Commons, https://commons.wikimedia.org/wiki/File:Chemical_synapse_schema_cropped.jpg). Electrical impulses propagate down axon fibers and trigger release of neurotransmitter-filled vesicles (inset) that diffuse across the synapse to bind and activate receptors on the postsynaptic cell. Astroglial cells (not shown) are variably interposed in the spaces between neurons. Glutamate transporters EAAT1/2 are found predominantly on glial cells and EAAT3 is expressed on neurons.

$[7,15,18,22,35]$. In addition, transporters aid in clearing synaptically released glutamate to facilitate rapid high-fidelity chemical communication between neurons $[12,38]$. While glutamate transporters are present on both neurons and glia, glial transporters are likely to mediate the bulk of glutamate uptake [9]. Disruption in their functions could lead to such problems as amyotrophic lateral sclerosis, Alzheimer's disease and neuronal death $[25,34]$.

The unitary turnover rate of transporters, i.e., the number of molecules that can be transferred from the extracellular space across the membrane by a single transporter per unit of time, is crucial for understanding of processes that shape synaptic transmission. The turnover rates reported in literature range from a few molecules per second on the lower end $[41,43]$ to the numbers that are several fold greater $[4,13]$. The chemical blockage of transporters is reported to rapidly elevate the ambient glutamate levels [7, 15, 18, 22, 35] and prolong glutamate receptor activation during synaptic transmission $[1,3,26,28,36]$. Paradoxically, the fastest turnover time constant of the predominant glutamate transporter in the brain was estimated to be nearly two orders of magnitude slower than the predicted time course of glutamate in the synapse $[8,37,43]$. It has been proposed that buffering of glutamate by transporters, rather than actual uptake, plays an important role in rapid lowering of neurotransmitter concentration during the first few milliseconds of synaptic transmission $[11,13]$. In this work 
we derive a model in order to reliably estimate turnover rates of EAAT1-3 from experimental data. We also develop a model combining spatial diffusion and transport that could help explain the sensitivity of postsynaptic glutamate receptor responses to modulation of uptake despite low turnover rates of transporters.

\section{EAATS CHEMICAL KINETICS MODELS}

\subsection{Existing models}

EAATs transport glutamate against its electrochemical gradient using the electrochemical gradients of other ions that are stoichiometrically coupled to glutamate flux. With each molecule of glutamate $3 \mathrm{Na}^{+}$and $1 \mathrm{H}^{+}$ are co-transported, and $1 \mathrm{~K}^{+}$is counter-transported [24,44], i.e., the net charge of +2 moves into the cell with each full cycle. This allows one to collect the data related to glutamate transport by recording the charge movement in cells expressing the transporters in electrophysiology experiments. The corresponding chemical kinetics model, depicted in Figure 2a, has been proposed and studied, e.g., in [5, 21]. Glutamate transporters also play a role of a chloride channel $[41,42]$, i.e., $\mathrm{Cl}^{-}$can permeate some states of the model depicted on Figure 2 a resulting in a net charge movement. The exact states that can interact in such a way with chloride ions are not known. If we assume that this is possible for all states, this will increase the number of states in the model to twice as many.

Unfortunately, the model is overparameterized with respect to the existing data and, therefore, most individual reaction rate constants have statistically unreliable estimates. It is possible to reduce the model under certain conditions during controlled experiments in order to extract important physical properties of the transporters, such as turnover rate. Let us consider an excised patch clamp type of experiments. A glass micropipette with very fine opening on one end $(\sim 1 \mu \mathrm{m}$ in diameter) is gently pushed against a single cell to form a tight seal with its membrane. Pulling the pipette causes rupture and reseal of the small portion of the membrane on the tip of the glass tube, a "patch". Depending on the technique of pulling, the outer side of the membrane could either face the inside of the pipette (called "inside-out patch") or the outside of the pipette (called "ouside-out patch"). The obtained patch retains the local structure of the cell membrane, including transmembrane proteins like ion channels and transporters. The solutions inside and outside of the micropipette are controlled by the investigator and represent cytoplasm and extracellular fluid. The solution in the bath, i.e., outside of the micropipette, can be rapidly switched by physically moving the pipette or bath. A piezo electric switch is often used for such purposes and it allows for a solution change within a few milliseconds. After the preparations one can study the flux of ions across the patch using electrophysiology techniques, e.g., voltage clamp (the membrane potential is fixed and the current that corresponds to the flow of ions is recorded). Since we have the total control of the solutions that represent intracellular and extracellular space in excised patch clamp experiments, let us adjust the ion concentrations in a such way that they accelerate the transitions of the model states in a clockwise manner. That is, increase sodium and hydrogen ions, and glutamate concentrations in the "extracellular space" to saturate the transporters with them; at the same time we reduce the potassium ion concentration in the "extracellular space" to facilitate $\mathrm{K}^{+}$unbinding from the transporter state $T_{o} K$; and do the opposite for the "intracellular space". The saturation of transporters with substrates will effectively eliminate some states of the model due to almost instantaneous binding of ligands to the transporters. Let us note that we are not concerned that the concentrations of compounds in the solutions will change due to the activity of transporters as the experiment goes on: the amount of molecules that can be transferred across a small patch is negligible compared to their initial concentrations in a time period it takes to perform the experiment. Under the described conditions the number of effective states in the model decreases to four. After addition of four more states that are responsible for conductance of chloride ions, the reduced model is obtained; see Figure $2 \mathrm{~b}$. Note that the model does not explicitly show concentrations of any compounds because they can be assumed to be fixed over the duration of the experiment and are included in the reaction rate constants. Similar models have been proposed and studied before, see e.g., [6, 13, 27, 41]. Although these smaller models seem to retain many, if not all, of the qualitative features of recorded currents, they still cannot be used for parameter estimation as they remain to be overparameterized with respect to the experimental data. 


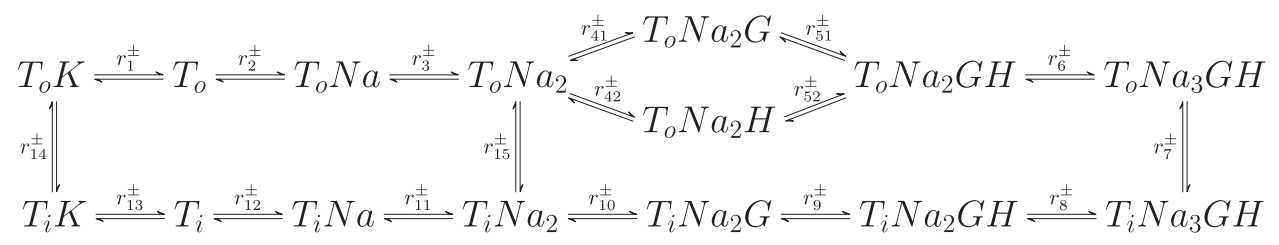

(a)

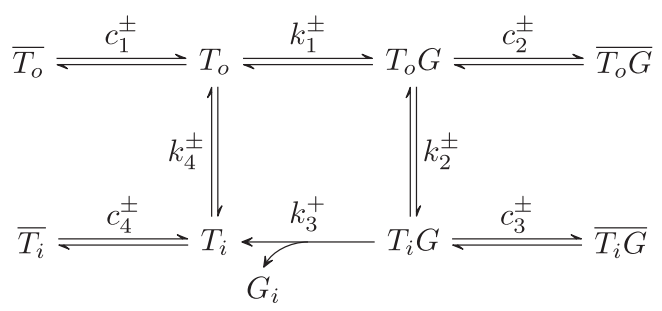

(b)

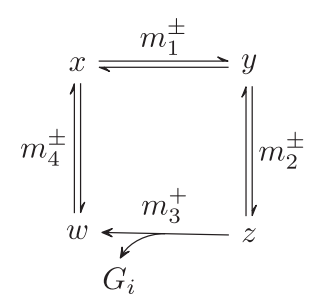

(c)

FiguRE 2. (a) 15-state model for EAATs. $T_{o}$ and $T_{i}$ stand for transporter facing extracellular and intracellular spaces, respectively; Na, K, H denote corresponding ions; G is L-glutamate; $r_{i}^{ \pm}$ for all $i$ are reaction rate constants. $r_{i}^{+}$and $r_{i}^{-}$correspond to a clockwise and counterclockwise directions, respectively. (b) The simplified 8-state model for the patch clamp experiment. The states with bars are the corresponding conducting states, which allow the flow of chloride ions.

(c) The chemical kinetics scheme that corresponds to the reduced model (2.4).

\subsection{Reduced model}

The chemical kinetics scheme depicted in Figure 2b can be represented by a system of differential equations using the rate law. For each state we define a corresponding variable, using the same notation, a fraction of all transporters observed to be in this state (e.g., the variable $T_{o}(t)$ is a portion of all transporters in $T_{o}$ state). The system has the following form:

$$
\begin{aligned}
\frac{\mathrm{d} T_{o}}{\mathrm{~d} t} & =c_{1}^{+} \overline{T_{o}}-c_{1}^{-} T_{o}-\left(k_{1}^{+}+k_{4}^{-}\right) T_{o}+k_{1}^{-} T_{o} G+k_{4}^{+} T_{i}, \\
\frac{\mathrm{d} T_{o} G}{\mathrm{~d} t} & =-c_{2}^{+} T_{o} G+c_{2}^{-} \overline{T_{o} G}-\left(k_{1}^{-}+k_{2}^{+}\right) T_{o} G+k_{1}^{+} T_{o}+k_{2}^{-} T_{i} G, \\
\frac{\mathrm{d} T_{i} G}{\mathrm{~d} t} & =-c_{3}^{+} T_{i} G+c_{3}^{-} \overline{T_{i} G}-\left(k_{2}^{-}+k_{3}^{+}\right) T_{i} G+k_{2}^{+} T_{o} G, \\
\frac{\mathrm{d} T_{i}}{\mathrm{~d} t} & =c_{4}^{+} \overline{T_{i}}-c_{4}^{-} T_{i}-k_{4}^{+} T_{i}+k_{3}^{+} T_{i} G+k_{4}^{-} T_{o}, \\
\frac{\mathrm{d} \overline{T_{o}}}{\mathrm{~d} t} & =-c_{1}^{+} \overline{T_{o}}+c_{1}^{-} T_{o}, \\
\frac{\mathrm{d} \overline{T_{o} G}}{\mathrm{~d} t} & =c_{2}^{+} T_{o} G-c_{2}^{-} \overline{T_{o} G} \\
\frac{\mathrm{d} \overline{T_{i} G}}{\mathrm{~d} t} & =c_{3}^{+} T_{i} G-c_{3}^{-} \overline{T_{i} G} \\
\frac{\mathrm{d} \overline{T_{i}}}{\mathrm{~d} t} & =-c_{4}^{+} \overline{T_{i}}+c_{4}^{-} T_{i} .
\end{aligned}
$$


During the experiments we will switch back and forth between the two bath mixtures: one will contain glutamate, and another will not, with all other substances being the same (see the next section for more details). All experiments start with no glutamate in the bath, which corresponds to an extracellular space in the outside-out patch experiments. The solution switch means an abrupt change in glutamate concentration. A transition of the transporter from $T_{o}$ state to $T_{o} G$ is impossible when the glutamate is absent, therefore $k_{1}^{+}$, the only coefficient that includes glutamate concentration, equals zero. Effectively, this means that every time the bath mixture is changed, we will use the end state of the system (2.1) with one value of $k_{1}^{+}$as initial conditions for the system (2.1) with another value of $k_{1}^{+}$(where $k_{1}^{+}$switches between a zero and a non-zero value).

As stated in the previous section, we cannot simply use the existing experimental data for estimating model parameters due to overparameterization of system (2.1). Therefore, some further model reduction is needed. According to [41], the transitions to conducting states are much faster compared to the reactions which correspond to glutamate transportation, i.e. $c_{i}^{ \pm} \gg k_{j}^{ \pm}$for all $i, j$. We can define a small parameter $0<\varepsilon \ll 1$ in the following way:

$$
c_{i}^{ \pm}=\widetilde{\widetilde{c_{i}^{ \pm}}}, \quad \widetilde{c_{i}^{ \pm}} \sim O(1), \quad k_{i}^{ \pm} \sim O(1), \quad i=\overline{1,4} .
$$

The boundary function method [39] allows for the reduction of system (2.1). We represent all the variables as asymptotic series with respect to the small parameter $\varepsilon$ :

$$
v(t)=v_{0}(t)+\Pi_{0} v(\tau)+\varepsilon\left(v_{1}(t)+\Pi_{1} v(\tau)\right)+\cdots,
$$

where $v(t)$ represents any variable in the system (2.1) and $\tau=t / \varepsilon$ is a rescaled (stretched) time variable. The terms in the asymptotic series that depend on $t$ are called the regular functions, and the terms that depend on $\tau$ are called the boundary functions. Next, we substitute (2.2) and (2.3) into the system (2.1). Equating coefficients of like powers of $\varepsilon$, separately for regular and boundary layer functions, we obtain the reduced model problems for different terms of the asymptotic expansion (2.3). The resulting system for the regular leading order approximation functions depends only on four variables, which we denote as $x(t), y(t), z(t)$, and $w(t)$ :

$$
\begin{aligned}
\frac{\mathrm{d} x}{\mathrm{~d} t} & =-m_{1}^{+} x+m_{1}^{-} y+m_{4}^{+} w-m_{4}^{-} x, \\
\frac{\mathrm{d} y}{\mathrm{~d} t} & =m_{1}^{+} x-m_{1}^{-} y-m_{2}^{+} y+m_{2}^{-} z, \\
\frac{\mathrm{d} z}{\mathrm{~d} t} & =m_{2}^{+} y-m_{2}^{-} z-m_{3}^{+} z, \\
\frac{\mathrm{d} w}{\mathrm{~d} t} & =m_{3}^{+} z-m_{4}^{+} w+m_{4}^{-} x,
\end{aligned}
$$

where

$$
\begin{array}{ll}
m_{1}^{+}=\frac{c_{1}^{+} k_{1}^{+}}{c_{1}^{+}+c_{1}^{-}}, & m_{3}^{+}=\frac{c_{3}^{-} k_{3}^{+}}{c_{3}^{-}+c_{3}^{+}}, \\
m_{1}^{-}=\frac{c_{2}^{-} k_{1}^{-}}{c_{2}^{-}+c_{2}^{+}}, & \\
m_{2}^{+}=\frac{c_{2}^{-} k_{2}^{+}}{c_{2}^{-}+c_{2}^{+}}, & m_{4}^{+}=\frac{c_{4}^{+} k_{4}^{+}}{c_{4}^{+}+c_{4}^{-}}, \\
m_{2}^{-}=\frac{c_{3}^{-} k_{2}^{-}}{c_{3}^{-}+c_{3}^{+}}, & m_{4}^{-}=\frac{c_{1}^{+} k_{4}^{-}}{c_{1}^{+}+c_{1}^{-}} .
\end{array}
$$


And all the functions of system (2.1) can be expressed through the functions of system (2.4) in a certain way. If we allow the system to reach its steady state before the first bath mixture change, then all the boundary layer functions will be identically zero and the leading order approximations for the variables in (2.1) will contain only the corresponding regular functions:

$$
\begin{aligned}
T_{o}(t) & =\frac{c_{1}^{+}}{c_{1}^{+}+c_{1}^{-}} x(t)+O(\varepsilon), & \overline{T_{o}}(t) & =\frac{c_{1}^{-}}{c_{1}^{+}+c_{1}^{-}} x(t)+O(\varepsilon), \\
T_{o} G(t) & =\frac{c_{2}^{-}}{c_{2}^{-}+c_{2}^{+}} y(t)+O(\varepsilon), & \overline{T_{o} G}(t) & =\frac{c_{2}^{+}}{c_{2}^{-}+c_{2}^{+}} y(t)+O(\varepsilon), \\
T_{i} G(t) & =\frac{c_{3}^{-}}{c_{3}^{-}+c_{3}^{+}} z(t)+O(\varepsilon), & \overline{T_{i} G}(t) & =\frac{c_{3}^{+}}{c_{3}^{-}+c_{3}^{+}} z(t)+O(\varepsilon), \\
T_{i}(t) & =\frac{c_{4}^{+}}{c_{4}^{+}+c_{4}^{-}} w(t)+O(\varepsilon), & \overline{T_{i}}(t) & =\frac{c_{4}^{-}}{c_{4}^{+}+c_{4}^{-}} w(t)+O(\varepsilon) .
\end{aligned}
$$

The initial conditions for system (2.4) have the form:

$$
\begin{aligned}
x(0) & =T_{o}(0)+\overline{T_{o}}(0), \\
y(0) & =T_{o} G(0)+\overline{T_{o} G}(0), \\
z(0) & =T_{i} G(0)+\overline{T_{i} G}(0), \\
w(0) & =T_{i}(0)+\overline{T_{i}}(0) .
\end{aligned}
$$

As mentioned earlier, all the experiments will start without glutamate in the bath. Thus, the initial conditions before the first switch correspond to the steady state in the absence of glutamate $\left(k_{1}^{+}=0\right)$ :

$$
\begin{aligned}
x(0) & =\frac{m_{4}^{+}}{m_{4}^{+}+m_{4}^{-}}, \\
y(0) & =0, \\
z(0) & =0, \\
w(0) & =\frac{m_{4}^{-}}{m_{4}^{-}+m_{4}^{+}} .
\end{aligned}
$$

The sum of the variables entering system (2.4) is constant and it equals to the sum of all the variables in system (2.1), which is 1 . It is possible to draw a chemical kinetics scheme that corresponds to the system (2.4) in the same manner as the scheme depicted in Figure $2 \mathrm{~b}$ relates to (2.1); see Figure 2c.

For each experiment the total current recorded during the experiment is a sum of the stoichiometric current (coupled flux of glutamate molecules and ions across the membrane), the conductive current due to the flow of chloride ions, and some constant leak current [27, 41]. One might expect that during the transportation of one molecule of glutamate +3 charge moves into the cell during the transition from state $T_{o} G$ to $T_{i} G\left(3 \mathrm{Na}^{+}, \mathrm{H}^{+}\right.$, and $\mathrm{Glu}^{-}$) and +1 charge during the transition from $T_{i} K$ to $T_{o}$ (just $\mathrm{K}^{+}$). However, the voltage-dependence of the transport suggests that the transporter also mediates a capacitive charge transfer (gating charge movement). This means that, although the net movement charge stays the same, actually the charge of +2 moves during the transition from state $T_{o} G$ to $T_{i} G$ with all other transitions having no immediate effect on the transportation current. The chloride-related current is proportional to the fractions of transporters observed in the conductive states $\left(\overline{T_{o}}, \overline{T_{o} G}, \overline{T_{i} G}\right.$, and $\left.\overline{T_{i}}\right)$. The resulting formula for the total current is:

$$
I=-A \cdot \overline{T_{o}}-B \cdot \overline{T_{o} G}-C \cdot \overline{T_{i} G}-D \cdot \overline{T_{i}}-E\left(k_{2}^{+} T_{o} G-k_{2}^{-} T_{i} G\right)+I_{\text {leak }} .
$$


Using (2.5), we can write the leading order approximation for the current $I_{0}$ that depends on $x, y, z$, and $w$. Since the system is in the steady state before the glutamate is introduced for the first time, i.e., in the state (2.6), we can subtract the steady state current in the above formula in order to identify the leading order approximation of the constant value of $I_{\text {leak }}$. In order to further reduce the number of parameters, we can express any one of the four variables in terms of the others since $x(t)+y(t)+z(t)+w(t)=1$ for any given instant of time $t$. We obtain the following formula:

$$
I_{0}(t)=-\mathcal{A} \cdot x(t)-\mathcal{B} \cdot y(t)-\mathcal{D} \cdot w(t)+\frac{\mathcal{A} m_{4}^{+}+\mathcal{D} m_{4}^{-}}{m_{4}^{+}+m_{4}^{-}}+O(\varepsilon),
$$

where

$$
\begin{aligned}
\mathcal{A} & =\frac{c_{1}^{-} A}{c_{1}^{+}+c_{1}^{-}}+\frac{c_{3}^{+} C-c_{3}^{-} k_{2}^{-} E}{c_{3}^{-}+c_{3}^{+}}, \\
\mathcal{B} & =\frac{c_{2}^{+} B+c_{2}^{-} k_{2}^{+} E}{c_{2}^{-}+c_{2}^{+}}+\frac{c_{3}^{+} C-c_{3}^{-} k_{2}^{-} E}{c_{3}^{-}+c_{3}^{+}} \\
\mathcal{D} & =\frac{c_{4}^{-} D}{c_{4}^{+}+c_{4}^{-}}+\frac{c_{3}^{+} C-c_{3}^{-} k_{2}^{-} E}{c_{3}^{-}+c_{3}^{+}} .
\end{aligned}
$$

Finally, the turnover rate of a particular transporter is equal to the influx of the glutamate molecules through the transportation cycle in the steady state:

$$
\begin{aligned}
\Theta & =\lim _{t \rightarrow \infty} \frac{\mathrm{d} G_{i}}{\mathrm{~d} t}=\lim _{t \rightarrow \infty} k_{3}^{+} T_{i} G(t) \\
& =\frac{k_{3}^{+}}{\frac{c_{4}^{+}+c_{4}^{-}}{c_{4}^{+}} \frac{k_{3}^{+}}{k_{4}^{+}}+\frac{c_{3}^{+}+c_{3}^{-}}{c_{3}^{-}}+\frac{c_{2}^{+}+c_{2}^{-}}{c_{2}^{-}} \frac{k_{3}^{+}+k_{2}^{-}}{k_{2}^{+}}+\left(\frac{c_{1}^{+}+c_{1}^{-}}{c_{1}^{+}}+\frac{c_{4}^{+}+c_{4}^{-}}{c_{4}^{+}} \frac{k_{4}^{-}}{k_{4}^{+}}\right) \frac{k_{2}^{+} k_{3}^{+}+k_{1}^{-} k_{3}^{+}+k_{1}^{-} k_{2}^{-}}{k_{1}^{+} k_{2}^{+}}} .
\end{aligned}
$$

Note, that although the concentrations of all the substrates are effectively constant for the settings of the described experiment, the turnover rate implicitly depends on glutamate concentration through the term $k_{1}^{+}$. Corresponding leading order approximation in terms of the parameters of the system (2.4) has the form:

$$
\Theta_{0}=\frac{m_{1}^{+} m_{2}^{+} m_{3}^{+} m_{4}^{+}}{m_{1}^{+} m_{2}^{+}\left(m_{3}^{+}+m_{4}^{+}\right)+m_{1}^{+} m_{4}^{+}\left(m_{2}^{-}+m_{3}^{+}\right)+\left(m_{4}^{+}+m_{4}^{-}\right)\left(m_{1}^{-} m_{2}^{-}+m_{1}^{-} m_{3}^{+}+m_{2}^{+} m_{3}^{+}\right)} .
$$

\subsection{Experiments and model fitting}

Stage V-VI Xenopus oocytes were injected with mRNA encoding one of EAAT1-3. We waited for 2-5 days to allow oocytes to express the transporters and excised outside-out patches after removing a vitelline membrane. The solution inside the pipette, which mimics intracellular space, contained $110 \mathrm{mM} \mathrm{KCl,} 3 \mathrm{mM} \mathrm{MgCl}_{2}$, $5 \mathrm{mM}$ Na-HEPES, and $10 \mathrm{mM}$ EGTA. The bath solutions, which correspond to extracellular space, contained $110 \mathrm{mM} \mathrm{NaCl}, 3 \mathrm{mM} \mathrm{MgCl} 2,5 \mathrm{mM} \mathrm{Na}-\mathrm{HEPES}$, and $10 \mathrm{mM}$ or no glutamate. All solutions were adjusted to pH 7.5 with Tris-base. Both bath mixtures flowed through a theta tube that was mounted on and controlled by a piezo electric switch. The voltage clamp method was used to record the flow of ions across the patch in a form of an electrical current, with the recording electrode in the pipette and the ground electrode in the bath. The voltage was fixed at $-60 \mathrm{mV}$, which is close to a natural resting potential of neurons and astrocytes. For more details of the voltage clamp technique see [41]. Each experiment started with the patch exposed to a bath solution with no glutamate and was allowed to stabilize and reach current steady state. After that we did a short rapid $50 \mathrm{~ms}$ step into a solution with glutamate before switching back to the no-glutamate mixture. Then 

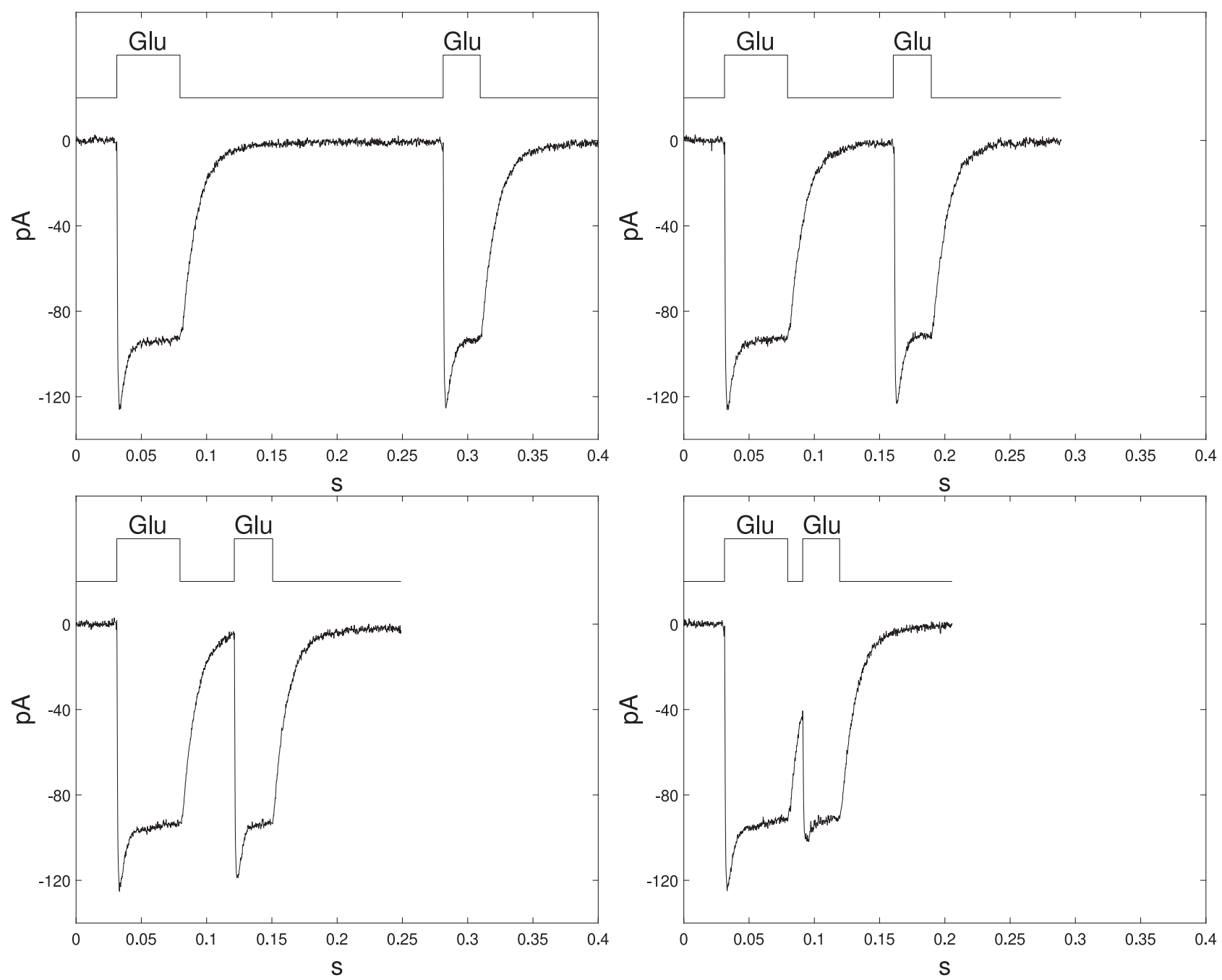

FiguRE 3. The currents recorded during the voltage clamp experiments in patches expressing EAAT1. The time courses of glutamate application are depicted as step functions above the actual data. Each application of the glutamate caused a spike of a negative (inward) current that relaxed to a new steady state. After the termination of the glutamate pulse, the system tended to its original steady state. The delays between the pulses depicted here are 200, 80, 40, and $10 \mathrm{~ms}$.

we waited for a varied controlled amount of time $(5,10,15,20,30,40,50,60,80,100,150,200,250$, and $300 \mathrm{~ms})$ and applied a second $30 \mathrm{~ms}$ step into the glutamate containing solution. Note that the only differences between the experimental trials was a delay after the first pulse. See Figure 3 for the representative data.

The data for each transporter were fitted to model (2.4), (2.6), and (2.7) using delayed rejection adaptive Metropolis Markov chain Monte Carlo (DRAM MCMC) method [14]. The fitting was done in MATLAB R2018a using MCMC toolbox. ${ }^{1}$ The fitted model solutions practically coincide with the experimental data for all cases, see Figure 4. The algorithm yielded empirical confidence regions for the parameters; the resulting MCMC chains

\footnotetext{
${ }^{\mathrm{I}} \mathrm{MCMC}$ toolbox for Matlab by Marko Laine http://helios.fmi.fi/ lainema/mcmc/
} 

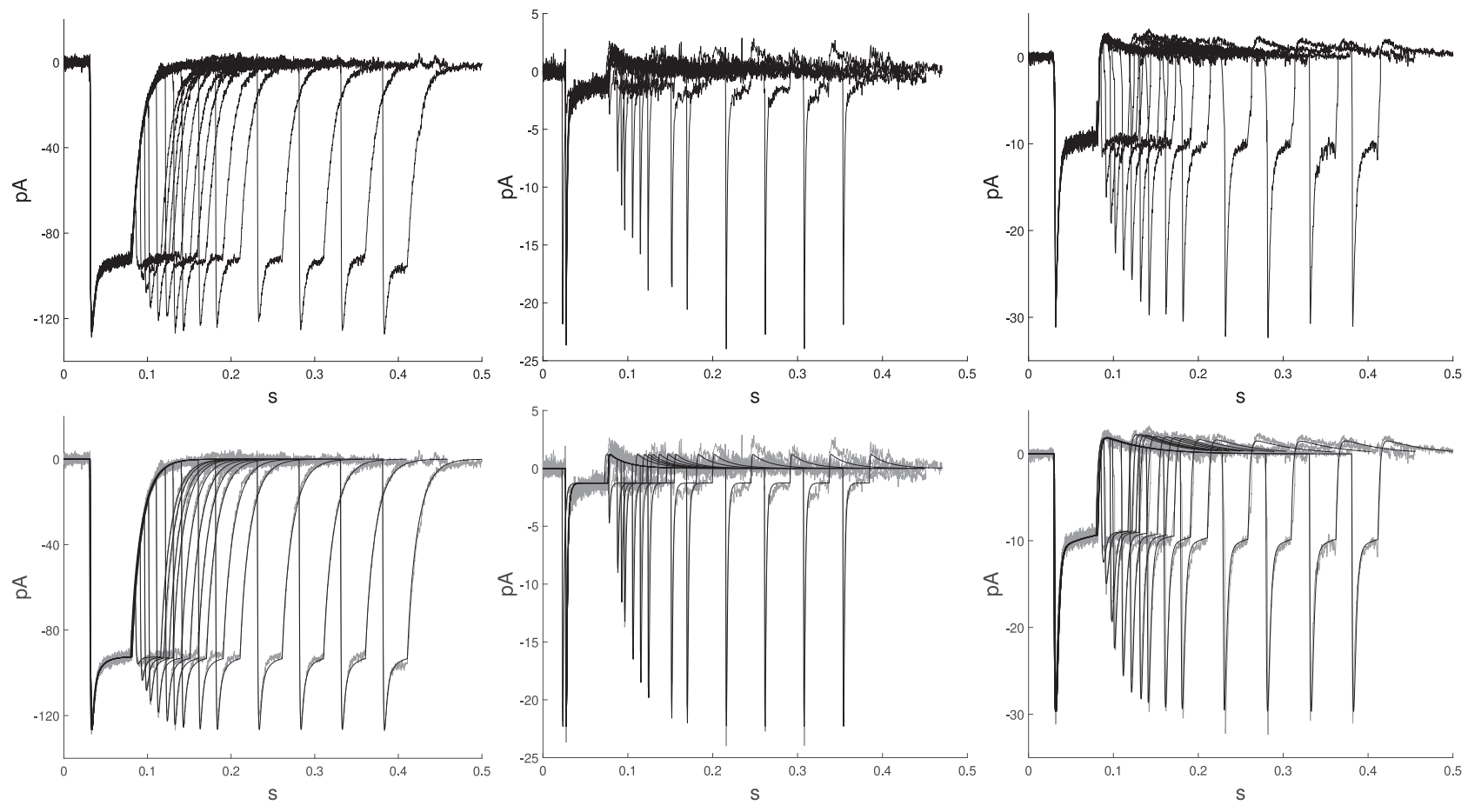

FiguRE 4. The currents recorded during the voltage clamp experiments in excised outside-out patches were fitted to the model. Top row depicts all the data for the corresponding transporter combined (left to right: EAAT1, EAAT2, EAAT3). All recorded pairs of pulses like the ones in Figure 3 were overlapped and the first pulses being almost identical. With the first pulse being fixed, it is easy to see the change in the dynamics of the second pulses, e.g., note how the peaks of the second pulses deteriorate but recover as the delay between the pulses increases. The second row shows the same data (now in gray) fitted using the model (black curves).

were used for turnover rate estimates and inferences using the formula (2.8); see Figures $5-7$ for confidence regions and Table 1 of fitted parameter values.

The estimated value for EAAT1 turnover rate found here $\left(15.45 \mathrm{~s}^{-1}, 99 \%\right.$ confidence interval $\left.[15.31,15.57]\right)$ is in agreement with previous studies: 16 molecules per second in EAAT1 [41]. The estimate for EAAT2 turnover rate $\left(23.96 \mathrm{~s}^{-1}, 99 \%\right.$ confidence interval $\left.[11.94,39.55]\right)$ is close to some previous studies, e.g., 14.6 molecules per second in [43], while it is lower than $\sim 100$ per second reported in [4]. The turnover rate of EAAT3 was reported to be much higher: about 100 molecules per second [13], compared to our estimate $\left(1.98 s^{-1}, 99 \%\right.$ confidence interval $[1.57,2.36])$.

\section{Diffusion MODEL}

EAAT turnover rates estimated here and in the other published studies are significantly slower than the estimated time course of glutamate in the synaptic cleft $[8,37]$. Nevertheless, the application of the glutamate transporter competitive antagonist DL-threo-benzyloxyaspartic acid (TBOA) to hippocampal slices were reported to rapidly elevate the ambient glutamate levels $[7,15,18,22,35]$. Moreover, the application of EAATs' blockers appears to prolong glutamate receptor activation during synaptic transmission $[1,3,26,28,36]$. While rise in ambient glutamate level is possible for any turnover rate given high enough density, the estimated EAATs concentrations in the synaptic cleft are too low to account for sufficiently fast changes that could reshape synaptic transmission [9]. It has been suggested that the initial fast phase of glutamate removal is due to diffusion 

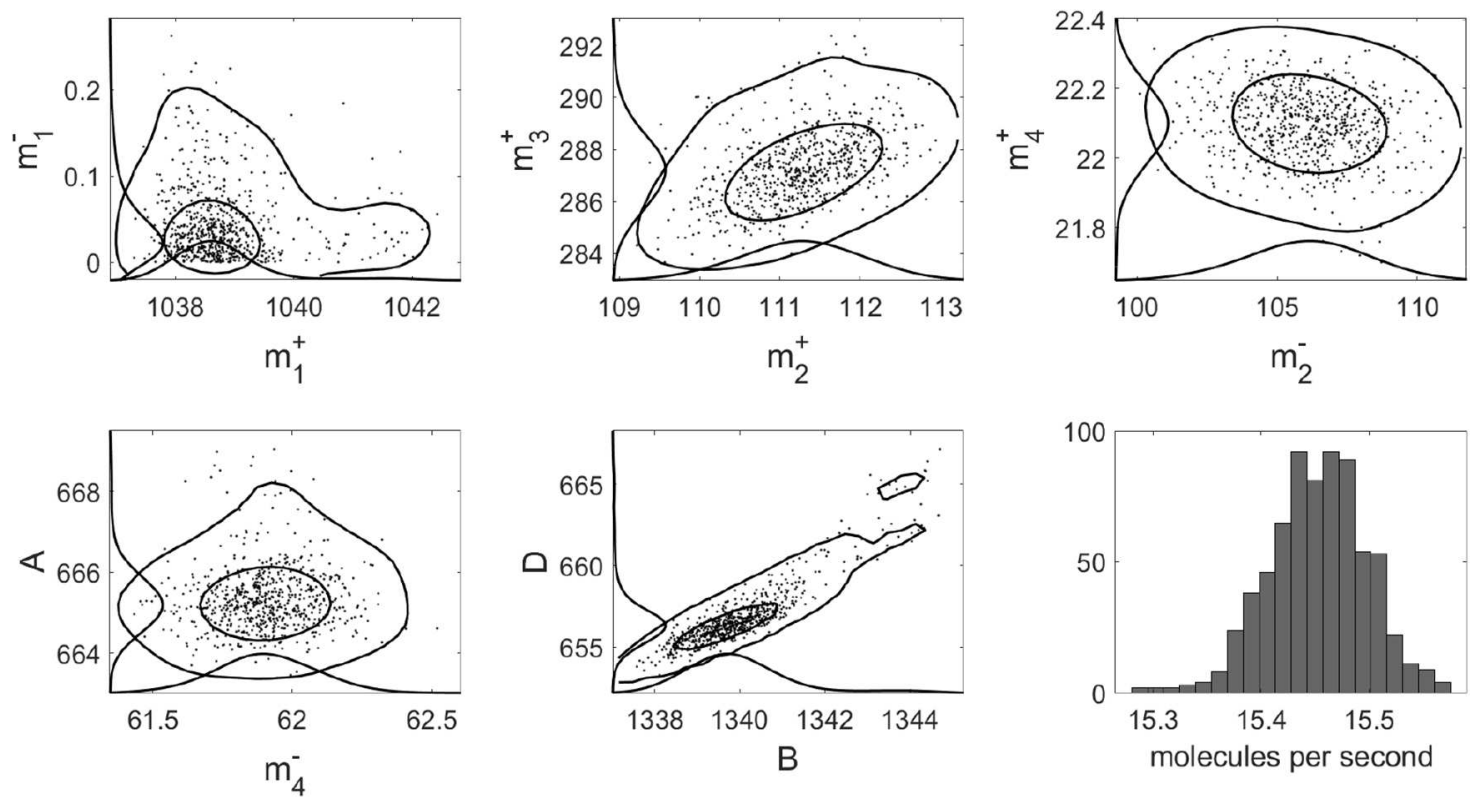

FiguRE 5. 95\% and 99\% confidence regions (inner and outer contours, respectively) for the model of EAAT1 yielded by the MCMC method. The last picture shows the distribution of EAAT1 turnover rate with the median value of $15.45 \mathrm{~s}^{-1}, 99 \%$ confidence interval: [15.31, 15.57].
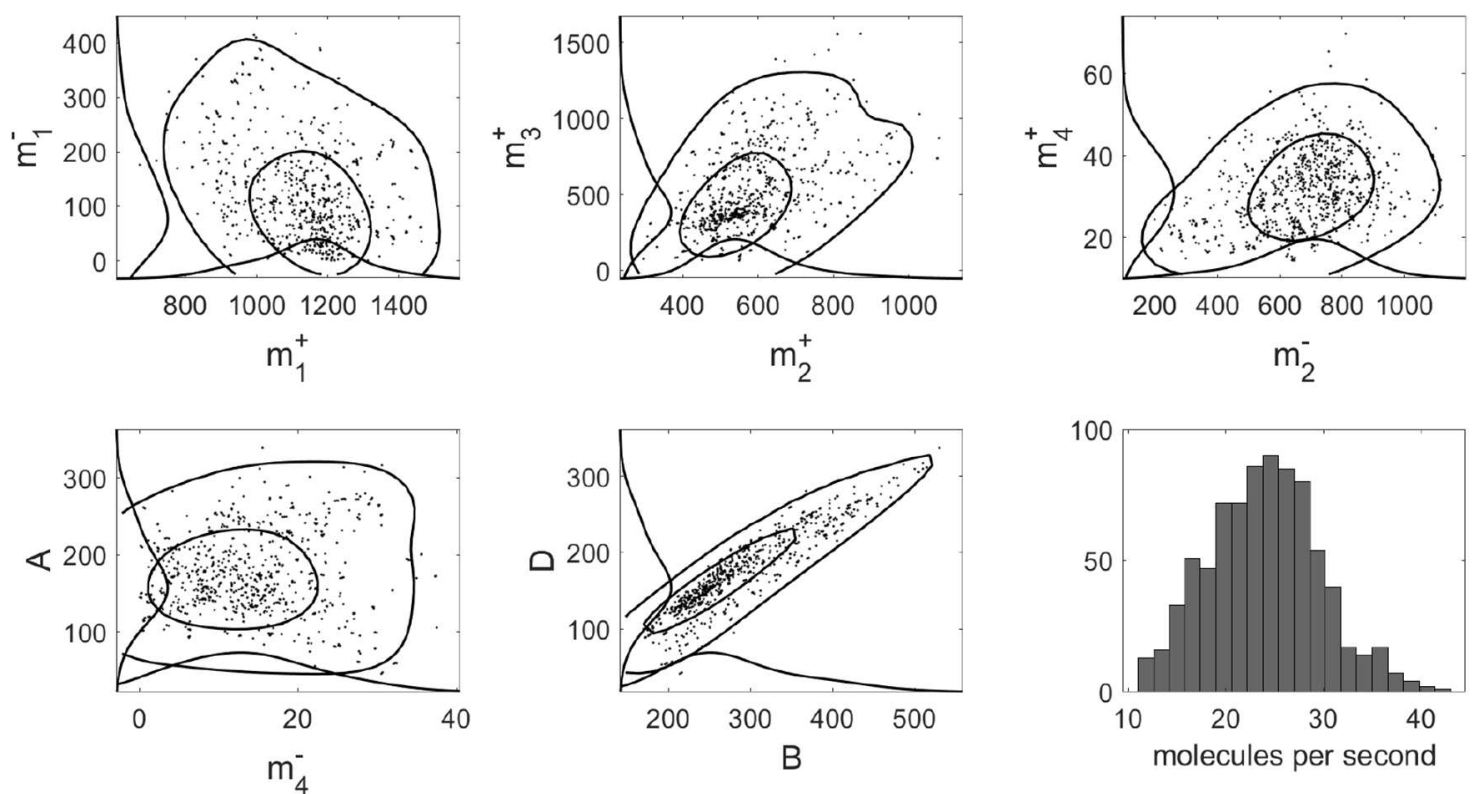

Figure 6. Similar results are shown for EAAT2. The median turnover rate value is $23.96 \mathrm{~s}^{-1}$, $99 \%$ confidence interval: [11.94, 39.55]. 

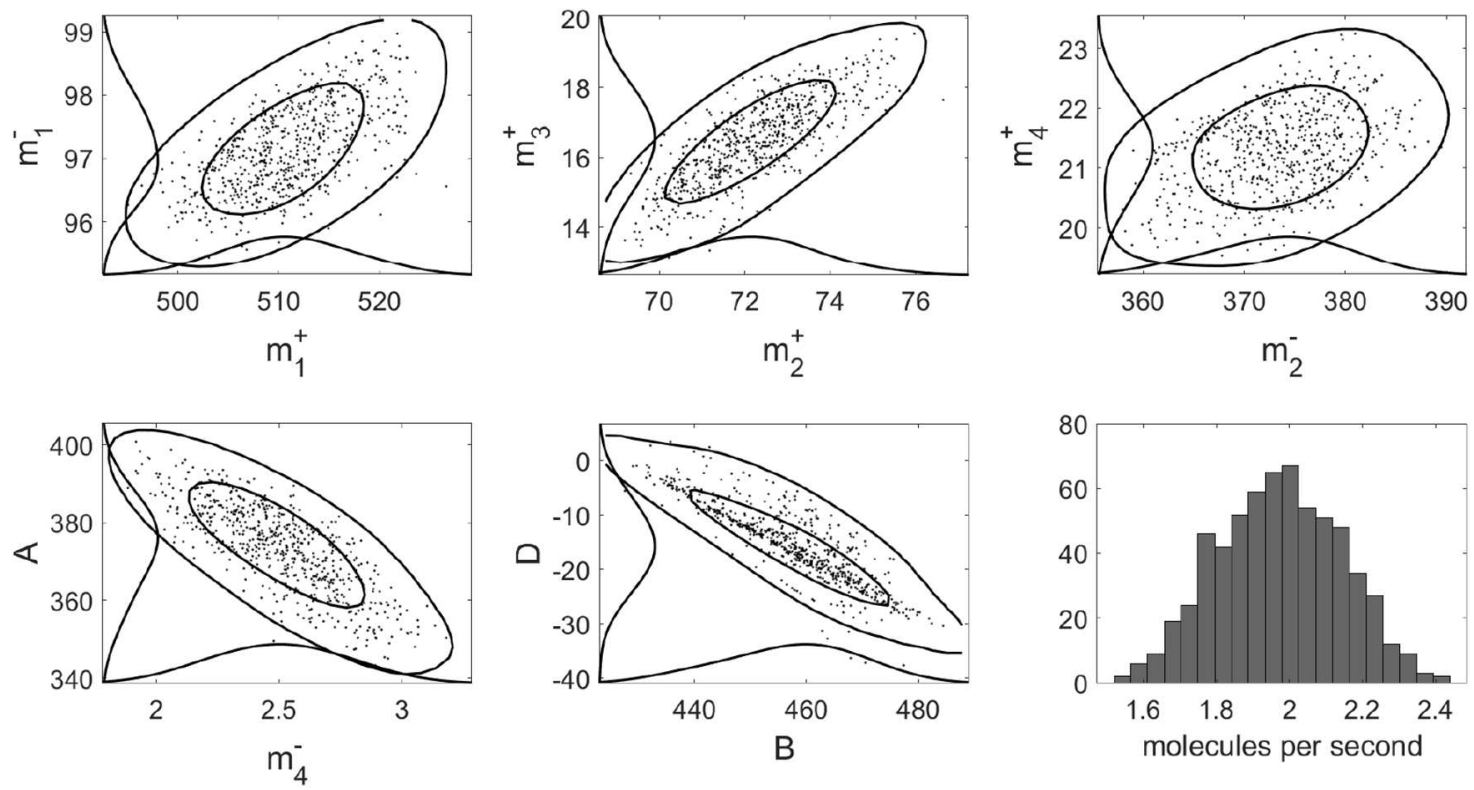

FIgURE 7. Similar results are shown for EAAT3. The estimated EAAT3 turnover rate of $1.98 \mathrm{~s}^{-1}, 99 \%$ confidence interval: $[1.57,2.36]$, was unexpectedly small compared to hundreds of molecules per second reported in other literature.

TABLE 1. All units are $\mathrm{s}^{-1}$.

\begin{tabular}{crlllllll}
\hline & \multicolumn{3}{c}{ EAAT1 } & & \multicolumn{2}{c}{ EAAT2 } & & \multicolumn{2}{c}{ EAAT3 } \\
\cline { 2 - 3 } \cline { 8 - 9 } \cline { 7 - 8 } & Median & $95 \%$ CI & & Median & $95 \%$ CI & & Median & $95 \%$ CI \\
\hline$m_{1}^{+}$ & 1038.65 & {$[1037.84 ; 1040.75]$} & & 1155.84 & {$[891.92 ; 1380.94]$} & & 510.72 & {$[501.70 ; 520.70]$} \\
$m_{1}^{-}$ & 0.03 & {$[0.00 ; 0.13]$} & & 101.99 & {$[12.78 ; 288.26]$} & & 97.10 & {$[96.12 ; 98.33]$} \\
$m_{2}^{+}$ & 111.25 & {$[110.10 ; 112.34]$} & & 556.67 & {$[413.85 ; 837.91]$} & & 72.22 & {$[70.05 ; 74.70]$} \\
$m_{2}^{-}$ & 106.11 & {$[102.67 ; 109.11]$} & & 697.34 & {$[359.99 ; 918.31]$} & & 373.72 & {$[362.31 ; 382.82]$} \\
$m_{3}^{+}$ & 287.23 & {$[285.15 ; 289.68]$} & & 422.36 & {$[142.80 ; 1027.13]$} & & 16.50 & {$[14.39 ; 18.38]$} \\
$m_{4}^{+}$ & 22.10 & {$[21.93 ; 22.25]$} & & 31.12 & {$[18.63 ; 45.44]$} & & 21.34 & {$[20.09 ; 22.45]$} \\
$m_{4}^{-}$ & 61.90 & {$[61.64 ; 62.17]$} & & 13.35 & {$[1.68 ; 28.93]$} & & 2.51 & {$[2.10 ; 2.90]$} \\
$A$ & 665.24 & {$[664.28 ; 666.78]$} & & 167.29 & {$[97.69 ; 266.50]$} & & 374.54 & {$[353.05 ; 390.47]$} \\
$B$ & 1339.80 & {$[1338.44 ; 1342.64]$} & & 265.48 & {$[196.36 ; 425.29]$} & & 458.20 & {$[436.92 ; 474.26]$} \\
$D$ & 656.43 & {$[654.81 ; 661.03]$} & & 163.93 & {$[94.32 ; 263.25]$} & & -15.56 & {$[-26.69 ;-3.76]$} \\
\hline
\end{tabular}

and buffering effect of transporters rather than the transport of neurotransmitter $[10,43]$. This buffering would require a relatively long lasting binding of glutamate molecule to the transporter $[13,23]$. It is worth noting that the data with prolongation of the receptor activation during synaptic transmission by blockers correspond to a whole cell or multiple cells rather than a single synapse $[1,3,26,28,36]$. Since the application of blockers raises ambient glutamate level which activates extrasynaptic receptors [22], it might play a significant role in apparent synaptic prolongation. In order to understand the relative contribution of transport and diffusion to dynamics of glutamate we created a simple spatial model of a single synapse, described below. We note that other approaches, e.g., Monte Carlo simulations, are also used for the analysis of neurotransmitter dynamics in a synaptic cleft; see, e.g., [32, 33]. 


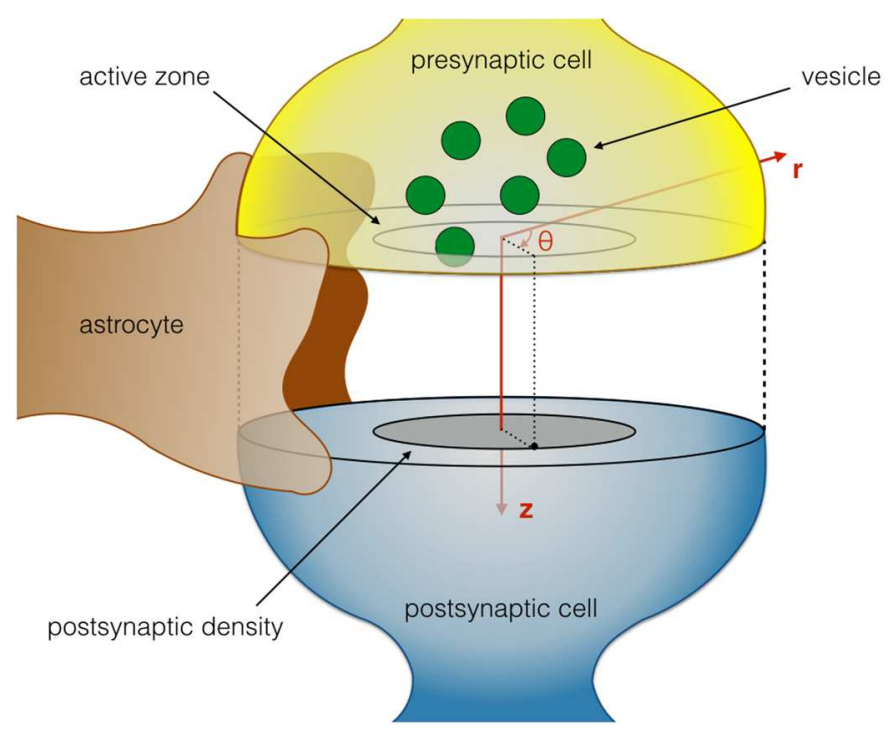

FIgURE 8. A cartoon representation of the synapse. The presynapic cell contains vesicles with glutamate that enter synaptic cleft through active zone (inner circle), diffuse towards the postsynaptic cell and are sensed by receptors located in the postsynaptic density (inner circle). Astrocyte envelops the cleft to some degree that varies from synapse to synapse. EAAT1-2 transporters are located on the astrocyte facing the synapse. EAAT2 also found in the cleft on the presynaptic cell outside of the active zone with expression $\sim 10$ times lower compared to the one in astrocytes. EAAT3 are expressed on the postsynaptic cell outside of the postsynaptic density.

Let us represent a synaptic cleft as a circular cylinder, with bases being pre- and postsynaptic cell surfaces, then we can describe the glutamate dynamics by a diffusion model. Upon arrival of an action potential, voltagegated calcium channels in the presynaptic cell axon terminal transiently open, resulting in a brief elevation of intracellular calcium and release of glutamate stored in intracellular vesicles. This release is mediated by a specialized region on a presynaptic cell, called the active zone, which contains protein that mediate calciumdependent vesicle fusion with the axon terminal membrane. The neurotransmitter diffuses across the synaptic cleft and reaches receptors on the postsynaptic cell, located in the region called the postsynaptic density (PSD). We assume that the active zone and PSD have a circular shape with their centers coinciding with the corresponding cylinder base centers. EAATs play an important role in removing glutamate from the synaptic cleft to terminate the signal and allow an effective information transduction between the cells as well as limit its negative effects (e.g., excitotoxicity). Some of EAATs are expressed in a synaptic cleft, while others are found on astrocytes: EAAT3 are exclusively expressed on neurons' in dendrites and somas and EAAT1 are found on astrocytes. EAAT2 are expressed on both astrocytes and synapses of presynaptic cells, although EAAT2's expression in synapses is about 10 times lower $[9,17]$. We assume that the distribution of EAATs is uniform where they are present. About $50 \%$ of the synapses in rat hippocampus region CA1 are enveloped by astrocytes and they are surrounded only partially [40]. This means that glutamate can escape synapse by either facilitated transport or free diffusion to the space outside the synapse. The latter is called a spillover and can lead to activation of neighboring synapses [40]. The model describes a single synapse, so we also assume no glutamate spillover from other synapses. See Figure 8 for a visual representation.

It is convenient to write a diffusion equation in cylindrical coordinates:

$$
\frac{\partial u}{\partial t}=D\left(\frac{1}{r} \frac{\partial}{\partial r}\left[r \frac{\partial u}{\partial r}\right]+\frac{1}{r^{2}} \frac{\partial^{2} u}{\partial \theta^{2}}+\frac{\partial^{2} u}{\partial z^{2}}\right), \quad t \geq 0, \quad 0<r \leq R, \quad 0 \leq \theta<2 \pi, \quad 0 \leq z \leq L,
$$


where $u(t, r, \theta, z)$ is a concentration of glutamate at a time $t$, at a radial distance $r$, angular coordinate $\theta$ and height $z ; D=0.4 \mu^{2} \mathrm{~ms}^{-1}$ is the diffusion coefficient of glutamate in a synaptic cleft, which is about half of that in a free medium $[29,32,45] ; R=150 \mathrm{~nm}$ is the radius of a synaptic cleft [32, 40]; $L=20 \mathrm{~nm}$ is the width of a synaptic cleft [32]. Let us note, that for $r=0$, the equation (3.1) can be rewritten in Cartesian coordinates at the origin. The boundary conditions are:

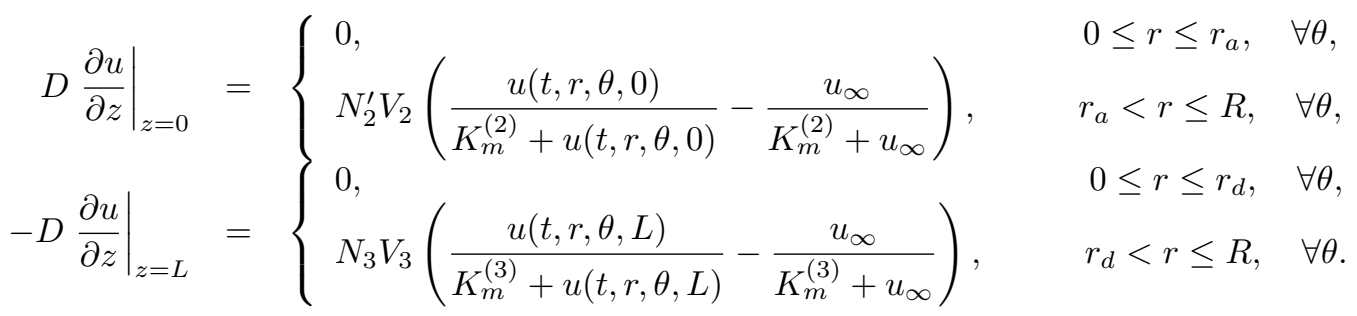

The last boundary condition is:

$$
-\left.D \frac{\partial u}{\partial r}\right|_{r=R}=\sum_{n=1}^{2} N_{n} V_{n}\left(\frac{u(t, R, \theta, z)}{K_{m}^{(n)}+u(t, R, \theta, z)}-\frac{u_{\infty}}{K_{m}^{(n)}+u_{\infty}}\right), \quad 0 \leq z \leq L, \quad 0 \leq \theta<2 \pi \rho,
$$

for the proportion of synapse covered by astrocyte, defined by the parameter $0 \leq \rho \leq 1$, and

$$
u(t, R, \theta, z)=u_{\infty}, \quad 2 \pi \rho \leq \theta<2 \pi
$$

Here $u_{\infty}=25 \mathrm{nM}$ is an ambient glutamate level $[7,15,18,22,35] ; r_{a}=100 \mathrm{~nm}$ is a radius of an active zone [31]; $r_{d}=100 \mathrm{~nm}$ is a radius of a postsynaptic density [32, 40]; $N_{1}=2300 \mu \mathrm{m}^{-2}, N_{2}^{\prime}=750 \mu \mathrm{m}^{-2}, N_{2}=7500 \mu \mathrm{m}^{-2}$, and $N_{3}=90 \mathrm{~mm}^{-2}$ are densities of EAAT1, EAAT2 on axon terminals, EAAT2 on astrocytes, EAAT3, respectively [9, 17]; $V_{1}=1.545 \times 10^{-2} \mathrm{~ms}^{-1}, V_{2}=2.396 \times 10^{-2} \mathrm{~ms}^{-1}$, and $V_{1}=0.198 \times 10^{-2} \mathrm{~ms}^{-1}$ are EAAT1-3 turnover rates, respectively. Glutamate uptake by the transporters is described by Michaelis-Menten kinetics with $K_{m}^{(1)}=20 \mu \mathrm{M}, K_{m}^{(2)}=18 \mu \mathrm{M}$, and $K_{m}^{(3)}=28 \mu \mathrm{M}$ constant for EAAT1-3, respectively [2, 9]. We note that the transporters are located on the surface of neurons and on the surface of an astrocyte, and for this reason the appearance of Michaelis-Menten kinetics in the boundary conditions is appropriate. The corresponding kinetics reactions take place in a thin layer near the boundary, and the boundary conditions (3.2), (3.3) are obtained by averaging of the processes taking place in the volume of the layer over the layer's thickness. The surface concentrations of the transporters are reported in the literature together with corresponding Michaelis-Menten constants values (see the numbers presented earlier). The averaging of the transporter concentrations over the height of the synaptic cleft as well as inclusion of Michelis-Menten kinetics as a reaction term in the diffusion equation may also be performed under certain conditions (see, $[19,20]$ for further discussion).

The astrocytes facilitate glutamate uptake and serve as a physical barrier to prevent a spillover. Due to complex brain environment the concentration of glutamate in the proximity of the synapse could vary, so the boundary condition (3.4) may not be adequate at all times. But since we are not considering a spillover phenomenon here, we assume it to be true.

Let us note that not every action potential in a presynaptic cell causes the release of even a single vesicle $[16,40]$, which contain about 3000 molecules $[30,32]$. To conservatively estimate the clearance of a synaptic cleft from the glutamate, we assume that exactly one vesicle is released on each stimulation from the center of the active zone. Thus, the initial conditions are

$$
u(0, r, \theta, z)=3000 \cdot \delta(r \cos \theta) \cdot \delta(r \sin \theta) \cdot \delta(z)+w(r, \theta, z)
$$


where $\delta(\cdot)$ is the Dirac delta function and $w$ is a state of the system before the pulse. $w(r, \theta, z) \equiv u_{\infty}$ for the first stimulation and for all consecutive ones $w(r, \theta, z)$ is equal to the end state of the model at the previous step.

We are interested in the dynamics of glutamate concentration on the surface of the PSD of the postsynaptic cell: the time it takes the glutamate to reach the receptors, how long the concentration stays high, how fast the glutamate gets cleared out from the cell surface to allow for adequate reaction to the consecutive signals. We used finite difference approximation for the derivatives in the right hand side of equation (3.1) and boundary conditions (3.2)-(3.3). Then, we numerically solved the resulting system of differential equations using MATLAB R2018a built-in solver ode15s. That allowed us to have an adaptive time step, which was desirable: the experimental data clearly show relatively fast and slow dynamics. The spatial grid used for the finite difference derivative approximation was the following: $r_{i}=i \cdot \Delta r \quad(i=\overline{0,30}, \Delta r=5 \mathrm{~nm}) ; \theta_{j}=j \cdot \Delta \theta \quad(j=\overline{0,29}, \Delta j=\pi / 15)$; $z_{k}=k \cdot \Delta z(k=\overline{0,4}, \Delta z=5 \mathrm{~nm})$. The simulations are depicted in Figure 9. Each vesicle release causes a sharp spike of glutamate concentration on the dendrite surface, which rapidly decays on a scale of a millisecond. The shape of the curve changes with the proportion $\rho$ of the synapse covered by an astrocyte. In the case when high frequency stimulation $(100 \mathrm{~Hz})$ was applied, the glutamate was cleared from the synaptic cleft between pulses even when $\rho>0.9$. Only when the synapse was completely covered by the astrocyte the solution drastically changed its behavior. In all the other cases the simulated time course of the glutamate simulated here was consistent with the experimental measurements reported in the literature $[8,37]$. However, let us note that it is unlikely that even a full astrocyte coverage of a synapse would be tightly sealed in reality [40]. We found that the change in EAAT3 turnover rate from about $2 \mathrm{~s}^{-1}$ to $110 \mathrm{~s}^{-1}$ (value reported in [13], data not shown) did not bear any noticeable effect due to the transporter low expression: only few transporters per synapse of a size modeled here, which in fact might be even lower [9]. The simulation of transporter blockage, i.e., setting the turnover rate of transporters to zero and rising the ambient glutamate level $u_{\infty}$ to the reported value [7, 18, 22], does not yield any significant change in the glutamate dynamics in the synaptic cleft. Therefore, we conclude that the blockage of the transporters could not significantly prolong the activation of synaptic glutamate receptors in an individual synapse.

\section{Conclusion}

EAATs actively remove glutamate from the extracellular space with an efficient ion gradient-coupling mechanism and the equilibrium thermodynamics of this transport mechanism result in nanomolar ambient levels that limit receptor activity over long time scales (for review see [35]). On the shorter time scales of pulsatile synaptic signaling, the kinetic properties of the transporters, such as the binding rates and turnover rates, are expected to influence the role transporters play in shaping the millisecond-scale time course of synaptically released glutamate. Estimates of these kinetic parameters vary in the literature [4, 13, 41, 43]. A precise understanding of these non-equilibrium kinetic parameters is important for understanding the role of transporters in shaping the dynamics of synaptic transmission. Here we used previously established chemical kinetics models of EAAT1-3 $[5,6,13,21,27,41]$ and derived a new model, the purpose of which was to avoid overparameterization problems in constraining turnover rates. The experiments were designed alongside the model to allow for such reduction. We used the model to fit experimental transporter data and estimate turnover rates of EAAT1-3. The values obtained for EAAT1-2 are in agreement with previous studies that used independent approaches [41, 43]. The turnover rate estimate obtained for EAAT3 in the present work was found to be about 100-fold lower than existing estimates [13]. In that study authors used a similar 6-state model to estimate turnover rate. Possible reasons for the discrepancy in estimates include overparameterization issues in the latter study. In any event, the overall effect of EAAT3 on synaptic transmission is expected to be smaller compared to the other transporter subtypes due to its lower density in brain [9] (but see [10]).

It is well-established that over longer time scales, transporters maintain low ambient glutamate levels, which rapidly rise upon pharmacological block [7, 15, 18, 22, 35]. We constructed a 3D diffusion model of a single synapse using estimated EAATs turnover rates and densities in order to formalize a simple proof of concept about the role of transporters at a single independent synapse. Our model simulation of the time course of 

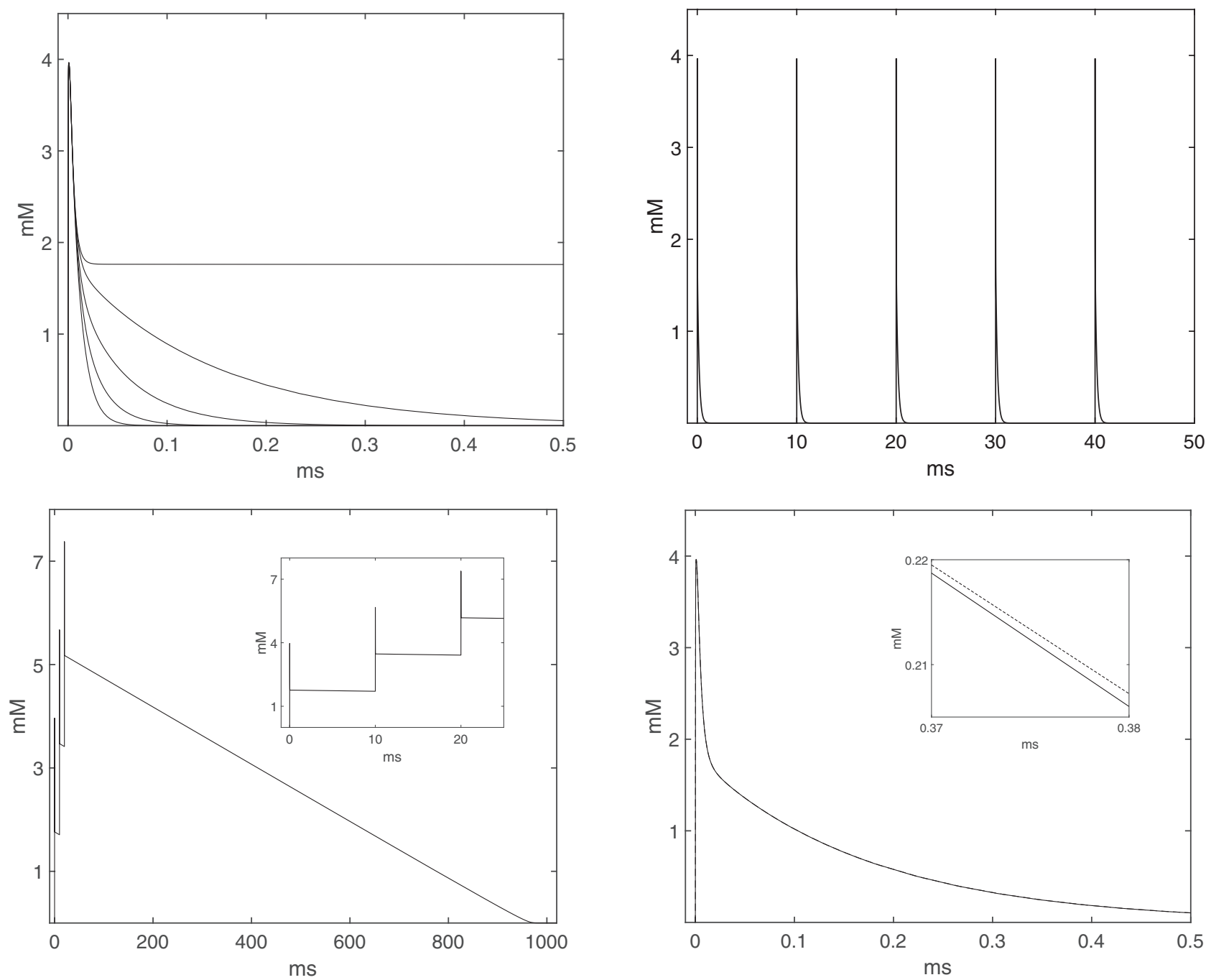

FiguRE 9. The dynamics of the average glutamate concentrations on the surface of the postsynaptic density "PSD" according to the diffusion model. Top left. Results of a release of a single vesicle into the synaptic cleft with an astrocyte covering various portions of the synapse. The corresponding parameter values of $\rho$ are 0 (fastest decay), 0.3, 0.6, 0.9, and 1 (slowest decay). When the astrocyte covers the synapse fully $(\rho=1)$ it takes much longer for the glutamate concentration to reach its steady state value $u_{\infty}$ (see also bottom left). Top right. Series of 5 high-frequency vesicle releases $(100 \mathrm{~Hz})$ with almost complete astrocytic envelopment $(\rho=0.95)$. Glutamate is cleared from the synaptic cleft and does not accumulate between the pulses. Bottom left. Series of 3 high-frequency vesicle releases $(100 \mathrm{~Hz})$ with complete astrocytic coverage $(\rho=1)$. Glutamate accumulates between the pulses and requires much longer time (almost $1 s$ here) to clear from the synaptic cleft via facilitated transport by EAATs. The inset graph shows the first $25 \mathrm{~ms}$ of the same plot that includes all three pulses. Bottom right. The modeling of transporter blockage in case $\rho=0.95$. The solid line represents the normal time course of glutamate (i.e., same as a single pulse from top right graph), the dashed line corresponds to the blockage case. The difference is almost impossible to see; hence the inset shows a zoomed portion of the same plot (note the axes scale). 
glutamate in the synaptic cleft is in good accordance with prior estimates [8,37]. Our findings support the idea that the rapid glutamate concentration rise and peak decay in the proximity of the postsynaptic receptors are achieved by the physical properties of the cleft itself, rather than active transport the transporters. According to the model, unless the synapse is completely sealed by an astrocyte, EAATs are not playing any significant role in removing synaptically released glutamate. This is not surprising, considering that time it takes for EAATs to transport one molecule of glutamate is several fold longer than the time the glutamate is present in the synapse at high concentrations. Although EAATs play a crucial role in controlling ambient glutamate levels, it is unlikely that EAATs shape the time course of synaptically released glutamate on the scale of a single synapse. A number of studies report that the pharmacological block of glutamate transporters prolongs receptor activation during synaptic transmission $[1,3,26,28,36]$. However, the data collected in these studies are on a scale of many synapses, or synapses with multiple release sites, which introduces additional phenomena, in particular pooling and heterosynaptic interactions that are not considered here. The results reported here are consistent with the idea that on the millisecond time scale of synaptic release, the role of transporters exclusively involves limiting activation of extrasynaptic receptors by spillover phenomena involving interaction of multiple synapses.

Acknowledgements. The authors thank Anastasios Tzingounis for data on Excitatory Amino Acid Transporter currents and the anonymous reviewers for their thoughtful comments, which led to substantial improvement of the quality of the paper.

\section{REFERENCES}

[1] N. Arnth-Jensen, D. Jabaudon and M. Scanziani, Cooperation between independent hippocampal synapses is controlled by glutamate uptake. Nat. Neurosci. 5 (2002) 325-331.

[2] J.L. Arriza, W.A. Fairman, J.I. Wadiche, G.H. Murdoch, M.P. Kavanaugh and S.G. Amara, Functional comparisons of three glutamate transporter subtypes cloned from human motor cortex. J. Neurosci. 14 (1994) 5559-5569.

[3] F. Asztely, G. Erdemli and D.M. Kullmann, Extrasynaptic glutamate spillover in the hippocampus: dependence on temperature and the role of active glutamate uptake. Neuron 18 (1997) 281-293.

[4] D.E. Bergles and C.E. Jahr, Glial contribution to glutamate uptake at Schaffer collateral-commissural synapses in the hippocampus. J. Neurophysiol. 18 (1998) 7709-7716.

[5] D.E. Bergles, A.V. Tzingounis and C.E. Jahr, Comparison of coupled and uncoupled currents during glutamate uptake by GLT-1 transporters. J. Neurosci. 22 (2002) 10153-10162.

[6] L. Borre, M.P. Kavanaugh and B.I. Kanner, Dynamic equilibrium between coupled and uncoupled modes of a neuronal glutamate transporter. J. Biol. Chem. 277 (2002) 13501-13507.

[7] P. Cavelier and D. Attwell, Tonic release of glutamate by a DIDS-sensitive mechanism in rat hippocampal slices. J. Physiol. 564 (2005) 397-410.

[8] J.D. Clements, R.A. Lester, G. Tong, C.E. Jahr and G.L. Westbrook, The time course of glutamate in the synaptic cleft. Science 258 (1992) 1498-1501.

[9] N.C. Danbolt, D.N. Furness and Y. Zhou, Neuronal vs glial glutamate uptake: resolving the conundrum. Neurochem. Int. 98 (2016) 29-45.

[10] J.S. Diamond, Neuronal glutamate transporters limit activation of NMDA receptors by neurotransmitter spillover on CA1 pyramidal cells. J. Neurosci. 21 (2001) 8328-8338.

[11] J.S. Diamond and C.E. Jahr, Transporters buffer synaptically released glutamate on a submillisecond time scale. J. Neurosci. 17 (1997) 4672-4687.

[12] J.S. Diamond and C.E. Jahr, Synaptically released glutamate does not overwhelm transporters on hippocampal astrocytes during high-frequency stimulation. J. Neurophysiol. 83 (2000) 2835-2843.

[13] C. Grewer, N. Watzke, M. Wiessner and T. Rauen, Glutamate translocation of the neuronal glutamate transporter EAAC1 occurs within milliseconds. PNAS 97 (2000) 9706-9711.

[14] H. Haario, M. Laine, A. Mira and E. Saksman, DRAM: efficient adaptive MCMC. Stat. Comput. 16 (2006) $339-354$.

[15] M.A. Herman and C.E. Jahr, Extracellular glutamate concentration in hippocampal slice. J. Neurosci. 27 (2007) $9736-9741$.

[16] N.A. Hessler, A.M. Shirke and R. Malinow, The probability of transmitter release at a mammalian central synapse. Nature 366 (1993) 569-572.

[17] S. Holmseth, Y. Dehnes, Y.H. Huang, V.V. Follin-Arbelet, N.J. Grutle, M.N. Mylonakou, C. Plachez, Y. Zhou, D.N. Furness, D.E. Bergles, K.P. Lehre and N.C. Danbolt, The density of EAAC1 (EAAT3) glutamate transporters expressed by neurons in the mammalian CNS. J. Neurosci. 32 (2012) 6000-6013.

[18] D. Jabaudon, K. Shimamoto, Y. Yasuda-Kamatani, M. Scanziani, B.H. Gähwiler and U. Gerber, Inhibition of uptake unmasks rapid extracellular turnover of glutamate of nonvesicular origin. Proc. Natl. Acad. Sci. USA 96 (1999) 8733-8738.

[19] L.V. Kalachev, Reduced model of neurotransmitter transport in the presence of generic receptors and transporters. J. Phys. Conf. Ser. 55 (2006) 114-129. 
[20] L.V. Kalachev, H.G. Kaper, T.J. Kaper, N. Popović and A. Zagaris, Reduction for Michaelis-Menten-Henri kinetics in the presence of diffusion. Proceedings of the 2006 International Conference in honor of Jacqueline Fleckinger. Electr. J. Differ. Equ. Conf. 16 (2007) 155-184.

[21] H.P. Larsson, A.V. Tzingounis, H.P. Koch and M.P. Kavanaugh, Fluorometric measurements of conformational changes in glutamate transporters. PNAS 101 (2004) 3951-3956.

[22] K. Le Meur, M. Galante, M.C. Angulo and E. Audinat, Tonic activation of NMDA receptors by ambient glutamate of non-synaptic origin in the rat hippocampus. J. Physiol. 580 (2007) 373-83.

[23] G.P. Leary, D.C. Holley, E.F. Stone, B.R. Lyda, L.V. Kalachev and M.P. Kavanaugh, The central cavity in trimeric glutamate transporters restricts ligand diffusion. Proc. Natl. Acad. Sci. USA 108 (2011) 14980-14985.

[24] L.M. Levy, O. Warr and D. Attwell, Stoichiometry of the glial glutamate transporter GLT-1 expressed inducibly in a Chinese hamster ovary cell line selected for low endogenous $\mathrm{Na}^{+}$-dependent glutamate uptake. J. Neurosci. 18 (1998) 9620-9628.

[25] C.L. Lin, L.A. Bristol, L. Jin, M. Dykes-Hoberg, T. Crawford, L. Clawson and J.D. Rothstein, Aberrant RNA processing in a neurodegenerative disease: the cause for absent EAAT2, a glutamate transporter, in amyotrophic lateral sclerosis. Neuron 20 (1998) 589-602.

[26] N.A. Lozovaya, M.V. Kopanitsa, Y.A. Boychuk and O.A. Krishtal, Enhancement of glutamate release uncovers spillovermediated transmission by N-methyl-D-aspartate receptors in the rat hippocampus. Neuroscience 91 (1999) 1321-1330.

[27] T.S. Otis and M.P. Kavanaugh, Isolation of current components and partial reaction cycles in the glial glutamate transporter EAAT2. J. Neurosci. 20 (2000) 2749-2757.

[28] T.S. Otis, Y.C. Wu and L.O. Trussell, Delayed clearance of transmitter and the role of glutamate transporters at synapses with multiple release sites. J. Neurosci. 16 (1996) 1634-1644.

[29] A.C.F. Ribeiro, M.M. Rodrigo, M.C.F. Barros, L.M.P. Verissimo, C. Romero, A.J.M. Valente and M.A. Esteso, Mutual diffusion coefficients of L-glutamic acid and monosodium L-glutamate in aqueous solutions at $T=298.15 \mathrm{~K}$. J. Chem. Thermodyn. 74 (2014) 133-137.

[30] N. Riveros, J. Fiedler, N. Lagos, C. Muñoz and F. Orrego, Glutamate in rat brain cortex synaptic vesicles: influence of the vesicle isolation procedure. Brain Res. 386 (1986) 405-408.

[31] D.A. Rusakov and D.M. Kullmann, Extrasynaptic glutamate diffusion in the Hippocampus: ultrastructural constraints, uptake, and receptor activation. J. Neurosci. 18 (1998) 3158-3170.

[32] L.P. Savtchenko and D.A. Rusakov, The optimal height of the synaptic cleft. PNAS 104 (2007) 1823-1828.

[33] A. Scimemi, H. Tian, J.S. Diamond, Neuronal transporters regulate glutamate clearance, NMDA receptor activation, and synaptic plasticity in the hippocampus. J. Neurosci. 29 (2009) 14581-14595. Erratum in J. Neurosci. 30 (2010) $9954-9956$.

[34] H.A. Scott, F.M. Gebhardt, A.D. Mitrovic, R.J. Vandenberg and P.R. Dodd, Glutamate transporter variants reduce glutamate uptake in Alzheimer's disease. Neurobiol. Aging 32 (2011) 553e1-553e11.

[35] W. Sun, D. Shchepakin, L.V. Kalachev and M.P. Kavanaugh, Glutamate transporter control of ambient glutamate levels. Neurochem. Int. 73 (2014) 146-151.

[36] W. Sun, K.M. Hoffman, D.C. Holley and M.P. Kavanaugh, Specificity and actions of an arylaspartate inhibitor of glutamate transport at the Schaffer collateral-CA1 pyramidal cell synapse. PLoS ONE 6 (2011) e23765.

[37] G. Tong and C.E. Jahr, Multivesicular release from excitatory synapses of cultured hippocampal neurons. Neuron 12 (1994) $51-59$.

[38] A.V. Tzingounis and J.I. Wadiche, Glutamate transporters: confining runaway excitation by shaping synaptic transmission. Nat. Rev. Neurosci. 8 (2007) 935-947.

[39] A.B. Vasil'eva, V.F. Butuzov and L.V. Kalachev, The boundary function method for singular perturbation problems. SIAM Studies in Applied Mathematics, Philadelphia, PA (1995).

[40] R. Ventura and K.M. Harris, Three-dimensional relationships between Hippocampal synapses and astrocytes. J. Neurosci. 19 (1999) 6897-6906.

[41] J.I. Wadiche and M.P. Kavanaugh, Macroscopic and microscopic properties of a cloned glutamate transporter/chloride channel. J. Neurosci. 18 (1998) 7650-7661.

[42] J.I. Wadiche, S.G. Amara and M.P. Kavanaugh, Ion fluxes associated with excitatory amino acid transport. Neuron 15 (1995) $721-728$.

[43] J.I. Wadiche, J.L. Arriza, S.G. Amara and M.P. Kavanaugh, Kinetics of a human glutamate transporter. Neuron 14 (1995) $1019-1027$

[44] N. Zerangue and M.P. Kavanaugh, Flux coupling in a neuronal glutamate transporter. Nature 383 (1996) 634-627.

[45] K. Zheng, T.P. Jensen, L.P. Savtchenko, J.A. Levitt, K. Suhling and D.A. Rusakov, Nanoscale diffusion in the synaptic cleft and beyond measured with time-resolved fluorescence anisotropy imaging. Sci Rep. 7 (2017) 42022. 